\title{
Próteses penianas no tratamento da disfunção erétil: a casuística de 13 anos
}

\author{
Luis Sepúlveda ${ }^{a, *}$, Ana Meireles ${ }^{b}$, Pedro Moreira $^{c}$, Henrique Dinis ${ }^{c}$, \\ Vera Marques ${ }^{c}$, Francisco Rolo ${ }^{c}$ e Alfredo Mota ${ }^{c}$
}

a Serviço de Urologia, Centro Hospitalar de Trás-os-Montes e Alto Douro (CHTMAD), Vila Real, Portugal
b Serviço de Cirurgia Plástica e Queimados, Centro Hospitalar Universitário de Coimbra (CHUC), Coimbra, Portugal
c Serviço de Urologia e Transplantação Renal, Centro Hospitalar Universitário de Coimbra (CHUC), Coimbra, Portugal

Recebido a 11 de novembro de 2014; aceite a 10 de outubro de 2016

Disponível na Internet a 3 de novembro de 2016

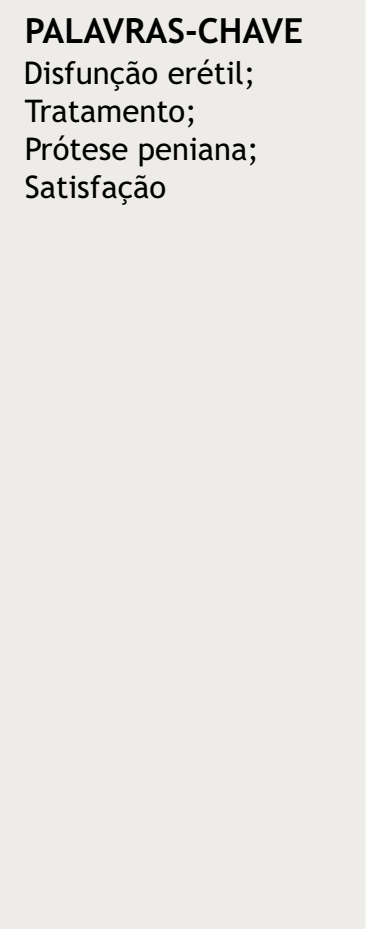

\begin{abstract}
Resumo
Introdução e objetivos: A colocação de próteses penianas (PP) é um tratamento comum e bem estabelecido na correção da disfunção erétil (DE) refratária ou com contraindicação ao tratamento médico. Embora seja a opção mais invasiva, apresenta altas taxas de satisfação dos doentes. $O$ objetivo deste estudo consistiu em avaliar os resultados cirúrgicos e a satisfação dos doentes, após a implantação de PP em 25 pacientes tratados no Centro Hospitalar Universitário de Coimbra (CHUC).

Material e métodos: Avaliámos 25 doentes com disfunção erétil (DE) orgânica submetidos a implantação de PP, insufláveis e semirrígidas, entre novembro de 2000 e novembro de 2013 . Os dados analisados foram obtidos a partir dos registos clínicos e entrevista telefónica, e englobavam a severidade clínica, comorbilidades, etiologia, tipo de PP, complicações cirúrgicas, necessidade de reintervenção e grau de satisfação do doente.

Resultados: Oitenta e oito por cento dos doentes apresentavam DE grave. As principais etiologias relatadas foram: multifatorial $(46,4 \%)$, arteriogénica (24\%) e neurológica (16\%). A maioria dos dispositivos implantados foram próteses do tipo insuflável (84\%). Em $80 \%$ dos procedimentos cirúrgicos não foram relatadas complicações, sendo que a taxa de reintervenção foi de $20 \%$. A percentagem de doentes satisfeitos/muito satisfeitos foi relativamente alta (65\%).

Discussão/conclusão: A colocação de PP continua a ser uma excelente alternativa para restaurar a função erétil nos doentes cujas terapias médicas falharam. Apesar do seu caráter invasivo, a implantação de PP demonstrou estar associada a uma baixa taxa de complicações e a alto grau de satisfação dos doentes.

(C 2016 Associação Portuguesa de Urologia. Publicado por Elsevier España, S.L.U. Este é um artigo Open Access sob uma licença CC BY-NC-ND (http://creativecommons.org/licenses/bync-nd/4.0/).
\end{abstract}

\footnotetext{
* Autor para correspondência.

Correio eletrónico: LuisSepulveda.Uro@gmail.com (L. Sepúlveda).
} 


\section{KEYWORDS}

Erectile dysfunction; Treatment; Penile prosthesis; Satisfaction

\section{Penile prosthesis in treatment of erectile dysfunction: 13-year experience}

\begin{abstract}
Introduction and Objectives: Penile prosthesis implantation is a common, well-established treatment for correcting medical refractory erectile dysfunction. Although more invasive than some of the other currently available therapies, PP surgery has the advantages of high patient satisfaction rates. The aim of this study was to evaluate the surgical results and patient satisfaction after implantation of PP in 25 patients treated at the University Hospital of Coimbra (CHUC).

Material and Methods: We evaluated 25 patients with erectile dysfunction who underwent implantation of PP, inflatable and semi-rigid, between November 2000 and November 2013. The information was obtained from medical records and telephone interviews and encompassed clinical severity, comorbidity, etiology, PP type, surgical complications, need for reintervention and degree of patient satisfaction.

Results: $88 \%$ of patients had severe ED. The main etiologies reported were: multifactorial (46.4\%), atherogenic (24\%) and neurologic (16\%). Most devices implanted were inflatable prostheses $(84 \%)$. In $80 \%$ of the cases there were no complications reported; the rate of re-intervention was $20 \%$. There were a high percentage of satisfied / very satisfied patients (65\%).

Discussion/Conclusion: PP surgery remains an excellent alternative in restoring erectile function in patients whose medical therapies have failed. Despite its invasive nature, the implementation of PP has proven to be associated with a low rate of complications and a high degree of patient satisfaction.

(c) 2016 Associação Portuguesa de Urologia. Published by Elsevier España, S.L.U. This is an open access article under the CC BY-NC-ND license (http: / / creativecommons.org/licenses/by-nc-nd/ $4.0 /)$.
\end{abstract}

\section{Introdução}

A primeira descrição conhecida sobre disfunção erétil (DE) surge no Papiro de Ebers, datado de 1.550 a.C, onde esta foi retratada como sendo «uma fraqueza do pénis» ${ }^{1}$. Atualmente, a DE é definida como a incapacidade em adquirir e/ou manter uma ereção adequada à realização de atividade sexual satisfatória ${ }^{2}$. A prevalência mundial da $D E$ moderada a severa é de aproximadamente $5-20 \%$, sendo que esta aumenta paralelamente com a idade ${ }^{2,3}$. Em Portugal, estima-se que cerca de $48,1 \%$ dos homens apresentem um grau variável de DE, com prevalências de 29,50 e $74 \%$, respetivamente para as faixas etárias dos 40-49 anos, 50-59 anos e 60-69 anos ${ }^{4}$.

A introdução dos inibidores da 5-fosfodiesterase revolucionou o tratamento para a $\mathrm{DE}$, constituindo atualmente a primeira linha terapêutica nestes doentes ${ }^{2,5,6}$. As opções de segunda linha incluem terapias intracavernosas injetáveis ou administração de prostaglandinas intraureterais. 0 tratamento cirúrgico representa a última opção terapêutica na maioria dos casos e envolve a colocação de próteses penianas (PP) e a reconstrução vascular do pénis (arterial ou venosa $)^{2,5-7}$.

As primeiras PP usadas no tratamento da DE datam de $1950^{1}$. Desde essa data tem-se observado uma evolução crescente na técnica cirúrgica e nas próteses utilizadas, desde próteses de peça única às próteses de 3 peças, próteses rígidas ou semirrígidas até às próteses hidráulicas. 0 principal impulso tecnológico ocorreu com o desenvolvimento da prótese insuflável de 3 peças em 1973, por Brantkey F. Scott, atualmente considerada a PP gold standard ${ }^{1,6}$. Hoje em dia existem no mercado 2 tipos de PP: as hidráulicas (insufláveis), melhores esteticamente e permitindo um aumento tanto em largura como em comprimento, e as semirrígidas, indicadas para doentes idosos ou com dificuldades motoras, caracterizadas pela sua excelente fiabilidade mecânica 2,6,7.

Esta evolução tecnológica tornou a cirurgia de PP uma boa alternativa terapêutica, com baixos riscos e bons índices de satisfação para os doentes ${ }^{2}$.

Este estudo pretendeu avaliar, retrospetivamente, os dados clínicos dos doentes submetidos a colocação de PP no Centro Hospitalar Universitário de Coimbra (CHUC), entre novembro de 2000 e novembro de 2013, relativamente à gravidade clínica, comorbilidades, causa da DE, tipo de prótese, complicações pós-operatórias, taxa de reintervenção e satisfação pós-operatória do doente.

\section{Materiais e métodos}

Análise retrospetiva dos dados clínicos dos doentes submetidos a colocação de PP, entre novembro de 2000 e novembro de 2013. Todos os doentes foram tratados cirurgicamente no CHUC. As variáveis analisadas foram: severidade clínica, comorbilidades, etiologia, tipo de PP, complicações cirúrgicas, necessidade de reintervenção, avaliação subjetiva do funcionamento da prótese e do grau de satisfação do doente. A variável etiologia foi dividida em: arteriogénica, neurológica, pós-prostatectomia e desconhecida. Quando presentes 2 ou mais fatores causais, a etiologia foi considerada multifatorial. No presente estudo optou-se por avaliar apenas 
as complicações mais frequentes (infeção, falência mecânica e erosão), dado que as complicações minor poderiam ser omissas no processo clínico.

Os dados foram recolhidos a partir dos processos clínicos e através de entrevistas telefónicas. Os doentes foram inquiridos quanto ao funcionamento da sua prótese e quanto ao grau de satisfação global, considerando em conjunto o funcionamento, a estética e as expectativas deste. Dada a dificuldade demonstrada, pela maioria dos doentes, em responder aos questionários Global Assessment Questionnaire (GAQ), Erectile Dysfunction Inventory of Treatment Satisfaction (EDITS) ou International Index of Erectile Function - satisfaction domain (IIEF), optou-se por pedir ao doente para atribuir um classificação para a variável funcionamento em ótimo, bom, razoável ou mau, e para a variável satisfação global em muito satisfeito, satisfeito, razoável ou insatisfeito.

Todos os participantes da amostra eram seguidos em consulta de Urologia do CHUC e apresentavam uma das seguintes condições: ineficácia, contraindicação ou efeitos adversos ao tratamento médico, terapêutica com vácuo insatisfatória ou ineficaz, falência orgânica, anomalias estruturais ou fibrose cavernosa.

A gravidade clínica foi caracterizada utilizando o «Massachusetts Male Aging Study (MMAS) Sexual Activity Questionnaire» e estratificada em 3 graus: leve, moderada ou grave.

Foram avaliadas as comorbilidades mais frequentes (hipertensão arterial, diabetes mellitus, coronariopatia e doença neurológica), tendo estas sido analisadas isoladamente ou agrupadas consoante a frequência da sua associação.

Para o processamento e análise dos dados coletados foi utilizado o programa estatístico IBM $®$ Statistical Package for Social Sciences (SPSS $®$ ) STATISTICS, versão 21.0. Os parâmetros referentes a caracterização da população foram analisados através de cálculo de média, mediana, desvio padrão e limites. As restantes variáveis (severidade clínica, comorbilidades, etiologia, tipo de PP, complicações cirúrgicas, necessidade de reintervenção, funcionamento e grau de satisfação) foram submetidas a análise descritiva.

\section{Resultados}

Entre novembro de 2000 e novembro de 2013, foram colocadas 25 PP a homens com DE orgânica. As idades variaram entre 28-70 anos, com idade média de 53,56 anos (tabela 1).

0 tempo médio de espera desde a primeira consulta no serviço de urologia/andrologia até à data da proposta

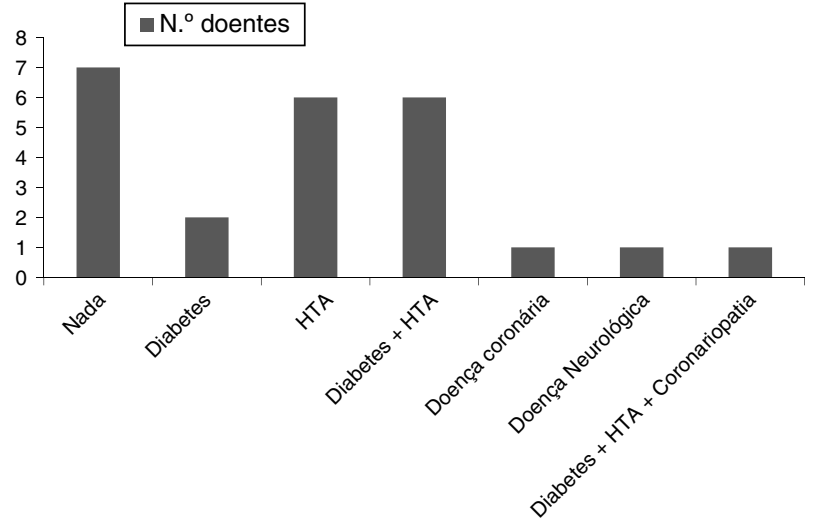

Figura 1 Distribuição das comorbilidades.

cirúrgica (intervalo $\mathrm{CP}$ ) foi de 32,52 meses e o tempo médio de espera desde a proposta cirúrgica até à data da intervenção cirúrgica (intervalo $\mathrm{PCx}$ ) foi de 16,42 meses (tabela 1).

A entrevista telefónica decorreu em junho de 2014: o tempo médio entre a data da cirurgia e a entrevista (intervalo $\mathrm{CXE}$ ) foi de 72,44 meses (tabela 1 ).

Oitenta e oito por cento $(22 / 25)$ dos doentes apresentavam queixas de $\mathrm{DE}$ grave e nenhum apresentava sintomatologia ligeira.

Em termos de comorbilidades, 7 doentes (28\%) não apresentavam nenhuma das comorbilidades avaliadas. A hipertensão arterial isolada (24\%) e em associação com diabetes mellitus (24\%) foram as comorbilidades mais frequentemente encontradas. Em $8 \%$ dos doentes a única comborbilidade identificada foi a diabetes mellitus. A doença coronária (4\%), a doença neurológica (4\%) e a associação diabetes mellitus, hipertensão arterial e coronariopatia (4\%) representaram as comorbilidades menos comuns (fig. 1). Não foi possível recolher a informação relativa a um doente.

A principal etiologia identificada foi multifatorial (40\%), seguida pela arteriogénica (24\%) e neurológica (16\%). Em 3 casos $(12 \%)$ a DE deveu-se a causa iatrogénica na sequência de prostatectomia radical e em 2 doentes (8\%) a causa da DE não era conhecida (fig. 2).

Ao longo dos 13 anos analisados, foram utilizados 4 tipos diferentes de PP: hidráulicas/insufláveis (modelo AMS 700® e Coloplast/Mentor TITAN $®$ ) e semirrígidas (modelo AMS Spectra ${ }^{\circledR}$ e modelo Coloplast Mentor Genesis $\left.®\right)$. As próteses insufláveis foram as mais utilizadas $(84 \%)$, respetivamente $52 \%$ para o modelo AMS $700 \circledast$ e $32 \%$ para o modelo Coloplast/Mentor TITAN® (tabela 2).

Tabela 1 Distribuição da amostra por idades, tempo médio de espera entre a primeira consulta e a proposta cirúrgica (intervalo $\mathrm{CP}$ ), entre a proposta cirúrgica e a cirurgia (intervalo $\mathrm{PCx}$ ) e entre a cirurgia e a entrevista telefónica (intervalo CxE)

\begin{tabular}{lcccr}
\hline & Idade (anos) & Intervalo CP (meses) & Intervalo PCx (meses) & Intervalo CxE (meses) \\
\hline Mínimo & 28 & 1 & 1 & 13 \\
Máximo & 70 & 142 & 132 & 163 \\
Média & 53,56 & 32,52 & 16,24 & 72,44 \\
Mediana & 59,00 & 18,00 & 10,00 & 60 \\
Desvio padrão & $\pm 12,142$ & $\pm 37,081$ & $\pm 25,545$ & $\pm 38,116$ \\
\hline
\end{tabular}


Tabela 2 Distribuição entre o tipo de prótese peniana e complicações pós-operatórias, funcionamento e grau de satisfação

\begin{tabular}{|c|c|c|c|c|c|}
\hline & AMS $700(13,52 \%)$ & AMS Spectra $(3,12 \%)$ & $\begin{array}{l}\text { Coloplast/ } \\
\text { Mentor TITAN } \\
(8,32 \%)\end{array}$ & $\begin{array}{l}\text { Coloplast/ } \\
\text { Mentor Genesis } \\
(1,4 \%)\end{array}$ & $\begin{array}{l}\text { Total } \\
\text { n (\%) }\end{array}$ \\
\hline \multicolumn{6}{|l|}{ Complicações } \\
\hline Não & 10 & 3 & 6 & 1 & $20(80)$ \\
\hline Falha mecânica & 2 & 0 & 1 & 0 & $3(12)$ \\
\hline Infeção & 1 & 0 & 0 & 0 & $1(4)$ \\
\hline Erosão & 0 & 0 & 1 & 0 & $1(4)$ \\
\hline \multicolumn{6}{|l|}{ Funcionamento } \\
\hline Ótimo & 4 & 0 & 2 & 0 & $6(30)$ \\
\hline Bom & 3 & 0 & 3 & 0 & $6(30)$ \\
\hline Razoável & 1 & 1 & 2 & 1 & $5(25)$ \\
\hline Mau & 2 & 0 & 1 & 0 & $3(15)$ \\
\hline \multicolumn{6}{|l|}{ Satisfação } \\
\hline Muito satisfeito & 4 & 0 & 3 & 0 & $7(35)$ \\
\hline Satisfeito & 3 & 0 & 3 & 0 & $6(30)$ \\
\hline Razoável & 0 & 1 & 1 & 1 & $3(15)$ \\
\hline Mau & 3 & 0 & 1 & 0 & $4(20)$ \\
\hline
\end{tabular}

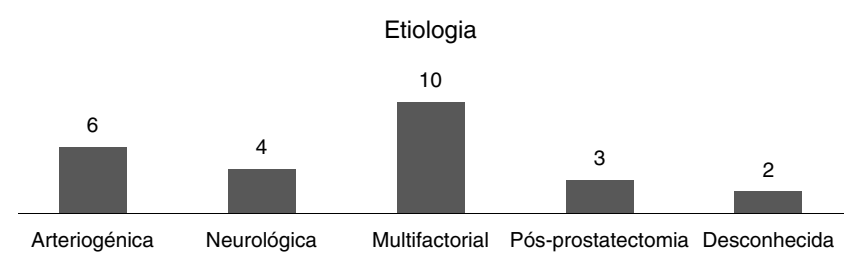

Figura 2 Distribuição da etiologia da disfunção erétil.

A principal complicação registada na nossa série foi a falha mecânica, presente em 3 casos (12\%), verificando-se iguais frequências entre a infeção e a erosão (um caso cada - 4\%). Em $80 \%$ dos casos não foram registadas quaisquer complicações pós-operatórias. A taxa de reintervenção foi de $20 \%$.

Apenas 20 doentes participaram na entrevista telefónica. Constatou-se que a maioria avaliou o funcionamento da sua prótese como ótimo ou bom (60\%). No entanto, uma percentagem apreciável classificou-o de uma forma negativa (mau, 15\%) (tabela 2). Em termos de grau de satisfação global, $65 \%$ afirmou encontrar-se muito satisfeito ou satisfeito com a sua PP, sendo que $20 \%$ relatava estar insatisfeito (tabela 2 ).

\section{Discussão}

Ainda não existe um método consolidado para a avaliação da gravidade clínica da $\mathrm{DE}$, o que a torna um processo não uniformizado.

Dois dos métodos mais amplamente utilizados para avaliar a severidade da DE são o IIEF e o Brief Male Sexual Function Inventory (BMSFI), ambos com enfoque sobre as áreas do desejo sexual, capacidade erétil, função sexual e satisfação geral ${ }^{3,8-10}$. A complexidade e elevado número de perguntas destes questionários levou à criação, por Derby et al., do «MMAS Sexual Activity Questionaire», que envolvia apenas uma pergunta. Os autores demonstraram uma alta correlação entre os resultados obtidos com o questionário de uma pergunta e os restantes questionários ${ }^{8}$. Neste estudo, a gravidade clínica foi avaliada segundo o MMAS: a elevada percentagem de doentes com queixas de DE grave (88\%) justifica-se pelo facto de o tratamento cirúrgico constituir a última linha de tratamento em doentes sem melhoras com as restantes atitudes terapêuticas.

Frequentemente, existem várias comorbilidades nos doentes com DE, sejam estes fatores causais ou agravantes da doença ${ }^{4}$. Neste estudo, o elevado número de doentes que não apresentavam comorbilidades (28\%) pode ser explicado pela baixa média de idades (53,56 anos) e por terem sido englobados doentes com DE pós-prostatectomia. A presença de múltiplas comorbilidades nos doentes com disfunção sexual e o aumento paralelo entre comorbilidades, idade e $\mathrm{DE}$, realça a preponderância do caráter multifatorial desta entidade clínica ${ }^{2,6,11}$.

Os principais fatores de risco para DE são a idade, patologia vascular, doenças metabólicas, doenças neurológicas e fármacos ${ }^{2,11}$. No nosso estudo, a causa mista $(41,7 \%)$ foi o principal fator etiológico da DE, semelhante ao que está demonstrado na literatura ${ }^{2,11}$. A causa arteriogénica foi responsável por aproximadamente $1 / 4$ dos casos. Nesta situação é essencial prestar especial atenção à sua relação com doença cardiovascular, podendo ser um indicador precoce da mesma ${ }^{11}$. A DE é uma das complicações pós-operatórias mais comuns da prostatectomia radical, com uma incidência de $25-75 \%^{2}$. A maioria dos doentes recupera a sua função sexual parcial ou totalmente em 12-24 meses, sendo improvável uma recuperação após $2 \operatorname{anos}^{12}$. Nos casos de DE refratária a tratamento médico após 2 anos da cirurgia, deverá ser ponderada a colocação de PP ${ }^{12}$.

No momento de escolha do tipo PP é preciso atender a diversos parâmetros, como a destreza manual e mental do doente, tamanho do cilindro e tamanho e localização do reservatório, bem como preço e expectativas do doente ${ }^{7,13}$. $A$ escolha do modelo passa pela preferência do cirurgião ${ }^{13}$. 
As próteses insufláveis apresentam excelentes resultados a longo prazo, assim como alto grau de satisfação do doente (70-98\%) e da parceira, sendo um dos dispositivos protésicos mais confiáveis e com maior sucesso na prática cirúrgica ${ }^{7,14}$. São habitualmente os dispositivos preferidos pelos doentes, pela sua estética mais natural ${ }^{2,5,15}$. As próteses semirrígidas são mais fáceis de manusear e apresentam menores taxas de falência mecânica7,13,15.

Dentro da amostra estudada, verificou-se que, à semelhança da literatura, as PP insufláveis foram as mais utilizadas (84\%).

As principais complicações pós-operatórias da colocação de PP são: infeção e a falência mecânica (mais comuns), problemas glandulares (encurtamento do pénis ereto ou encurvamento da porção distal do pénis), deslocamento do reservatório, autoinsuflação, erosão ou extrusão do cilindro distal (por perda de sensibilidade) ou fibrose cavernosa $2,7,13,16$. A infeção é a complicação mais grave, impondo na maioria dos casos a remoção imediata do dispositivo. Normalmente decorre por contaminação intraoperatória pela flora normal da pele (Staphylococcus epidermis - agente etiológico mais comum) ${ }^{13,15,17}$. Na literatura a taxa de infeção ronda os $2-3 \%$, sendo que alguns autores relatam taxas de infeção de até $16 \%^{2,13,16}$. Estas frequências são sobreponíveis à verificada neste estudo (4\%). A incidência da falência mecânica varia dentro de cada estudo e de acordo com o tipo de prótese, tendo-se verificado um decréscimo nas últimas décadas. As atuais incidências de falência mecânica e de erosão são de aproximadamente $5 \%$, ligeiramente superior às constatadas nesta amostra $(4 \%)^{2,5}$. As taxas de erosão descritas nas próteses semirrígidas são ligeiramente superiores ${ }^{13,15}$. A reintervenção ocorre geralmente por mal funcionamento ou infeção da prótese. Doentes que são submetidos a várias reparações referem uma diminuição do tamanho do pénis e aumento das complicações infeciosas, com subsequente aumento do grau de insatisfação ${ }^{14,17}$. Na amostra estudada, a maioria dos doentes não necessitaram de uma segunda intervenção (80\%), o que é um resultado bastante positivo, tendo em conta as dificuldades técnicas e riscos de insucesso inerentes a uma segunda abordagem cirúrgica ${ }^{13}$.

De todos os tratamentos disponíveis para a DE, as PP apresentam a maior taxa de satisfação, tanto do doente como da parceira, embora seja o método menos escolhido pelo seu caráter invasivo ${ }^{7,14,16}$. Dentro das PP, verifica-se que existem maiores índices de satisfação para as próteses insufláveis ${ }^{7,14}$.

Dois grandes fatores para a satisfação do doente são a rapidez em obter uma ereção e a rigidez consecutivamente obtida $^{14}$. Foram propostos vários questionários para avaliar a satisfação do doente, GAQ, EDITS, IIEF, bem como diferentes variáveis, como tamanho final do pénis, complicações cirúrgicas, funcionamento da prótese e cumprimento das expectativas $^{9,14}$.

Neste estudo, a satisfação do paciente foi avaliada de uma forma global, independentemente do tipo de prótese colocada. Baseou-se na opinião subjetiva do doente, tendo em consideração as variáveis acima citadas e a facilidade de manuseamento, similaridade com a atividade sexual prévia e a vertente estética. Como referido anteriormente, na maioria dos estudos o grau de satisfação dos doentes e da parceira é alto (70-98\%), bastante superior ao apurado neste estudo (satisfação, 65\%; funcionamento da PP ótimo/bom, 60\% $)^{2,7,13,14,16}$. Paralelamente, o índice de insatisfação relatado na literatura varia entre $0-8 \%$, consideravelmente inferior ao verificado neste estudo (insatisfação $20 \%$; mau funcionamento da PP $15 \%)^{14}$.

Estas diferenças poderão estar relacionadas com o reduzido tamanho da amostra em estudo, com a subjetividade do inquérito realizado (baseado na classificação global atribuída pelo doente), assim como com o excessivo tempo decorrido entre a colocação da prótese e a realização do questionário de satisfação, nalguns casos superior a 12 anos. Nestas situações a avaliação da satisfação estará sempre dependente e enviesada pela deterioração progressiva da prótese, assim como pelo próprio envelhecimento do doente.

\section{Conclusão}

A colocação de PP representa a última linha terapêutica no tratamento da $\mathrm{DE}$, sendo o único tratamento definitivo e com excelentes resultados a longo prazo. Embora seja um método invasivo, apresenta uma taxa relativamente pequena de complicações e elevada satisfação do doente.

Têm sido feitos inúmeros avanços nesta área e, dentro do CHUC, a experiência inicial é satisfatória. Os resultados são sobreponíveis aos apresentados na literatura para a maioria dos parâmetros avaliados, sendo que, com o progressivo aumento da experiência cirúrgica, seja previsível uma melhora gradual.

As principais limitações a este estudo são o seu caráter retrospetivo, a subjetividade do inquérito de satisfação realizado e o reduzido número de doentes num período de tempo relativamente longo, atribuível ao facto de ser uma casuística de um único serviço de urologia. Seria importante a comparação com outros serviços hospitalares pelos subsequentes benefícios decorrentes da troca de informação, um veículo essencial à evolução do conhecimento médico.

\section{Responsabilidades éticas}

Proteção de pessoas e animais. Os autores declaram que para esta investigação não se realizaram experiências em seres humanos e/ou animais.

Confidencialidade dos dados. Os autores declaram ter seguido os protocolos do seu centro de trabalho acerca da publicação dos dados de pacientes.

Direito à privacidade e consentimento escrito. Os autores declaram que não aparecem dados de pacientes neste artigo

\section{Conflito de interesses}

Os autores declaram não haver conflito de interesses.

\section{Referências}

1. Jara J, Lledó E. Historical approach to the surgical treatment of erectile dysfunction. Actas Urol Esp. 2013;37:445-50. 
2. Hatzimouratidis K, Amar E, Eardley I, Giuliano F, Hatzichristou D, Montorsi F, et al. Guidelines on male sexual dysfunction: Erectile dysfunction and premature ejaculation. Eur Urol. 2010; $57: 804-14$.

3. Morales J, Rolo F. Epidemiologia da disfunção erétil: revisão de literatura. Acta Urol Port. 2001;18:31-4.

4. Teles AG, Carreira M, Alarcão V, Sociol D, Aragüés JM, Lopes $L$, et al. Prevalence, severity, and risk factors for erectile dysfunction in a representative sample of 3,548 portuguese men aged 40 to 69 years attending primary healthcare centers: Results of the Portuguese erectile dysfunction study. J Sex Med. 2008;5:1317-24.

5. Siddiqi K, Lewis RW. Surgical therapy for the treatment of erec tile dysfunction. Nat Clin Pract Urol. 2008;5:174-5.

6. Carreño Rodríguez J, Martínez Abreu J, Toledo Viera A. Clinical evolution in patients with penile prosthesis implant. Arch Esp Urol. 2009;62:466-72.

7. Hellstrom WJG, Montague DK, Moncada I, Carson C, Minhas S, Faria G, et al. Implants, mechanical devices, and vascular surgery for erectile dysfunction. J Sex Med. 2010;7 1 Pt 2:501-23.

8. Derby CA, Araujo AB, Johannes CB, Feldman HA, McKinlay $J B$. Measurement of erectile dysfunction in population-based studies: The use of a single question self-assessment in the Massachusetts Male Aging Study. Int J Impot Res. 2000;12:197-204.
9. Rosen RC, Cappelleri JC, Gendrano N. The International Index of Erectile Function (IIEF): A state-of-the-science review. Int J Impot Res. 2002;14:226-44.

10. O'Leary MP, Rhodes T, Girman CJ, Jacobson DJ, Roberts RO, Lieber MM, et al. Distribution of the Brief Male Sexual Inventory in community men. Int J Impot Res. 2003;15:185-91.

11. Grant P, Jackson G, Baig I, Quin J. Erectile dysfunction in general medicine. Clin Med. 2013;13:136-40.

12. McCullough AR. Prevention and management of erectile dysfunction following radical prostatectomy. Urol Clin North Am. 2001;28:613-27.

13. Evans $C$. The use of penile prostheses in the treatment of impotence. Br J Urol. 1998;81:591-8.

14. Trost LW, Baum N, Hellstrom WJG. Managing the difficult penile prosthesis patient. J Sex Med. 2013;10:893-906.

15. Sadeghi-Nejad H. Penile prosthesis surgery: A review of prosthetic devices and associated complications. J Sex Med. 2007;4:296-309.

16. Mulcahy JJ, Austoni E, Barada JH, Choi HK, Hellstrom WJ, Hellstrom WJG, et al. The penile implant for erectile dysfunction. J Sex Med. 2004;1:98-109.

17. Selph JP, Carson CC. Penile prosthesis infection: Approaches to prevention and treatment. Urol Clin North Am. 2011;38: 227-35. 